\title{
CURRENCY RECOGNITION SYSTEM USING IMAGE PROCESSING
}

\author{
Shivam Singh, Anshuman Tiwari, Sameer Shukla, Sankalp Pateriya \\ Department of Computer Science and Engineering \\ Student, IMS Engineering College \\ Ghaziabad, India
}

\begin{abstract}
In this paper, we invented a system for currency identification using a technique called image processing. The suggested approach can be used for identifying the nation (country) or origin and the cost or the denomination of a given note as well. We have taken simplest paper currencies into consideration. This system works by first pointing the nation of origin using assured existing region of interest, and then extricating the cost value using attributes like color, text, or size of the currency, based on how much the notes in the same nation varies. We have examined some of the most popular currencies, along with their cost value or denominations. Our system is able to accurately and expeditiously classify sample notes.
\end{abstract}

Keywords - Machine Learning, Currency detection, Feature extraction, Neural Network.

\section{INTRODUCTION}

As per the survey conducted, there exist more than 150 different currencies in the world presently. These currencies varies substantially in characteristics such as color, size and shape. Unlike the ancient times, the business and exchange among various nations has expanded in all varieties of levels. The need for acquiring knowledge about approximately all of the currencies by means of the banks has been extraordinarily critical. However for any human being it is almost impossible to recognize or memorize all the types of currencies regulating throughout the world. Thus it becomes necessity to have an efficient automatic system that helps in recognizing paper currency which is pivotal for the future. In this paper, we suggest an automated system for currency recognition using Image processing techniques. Our system works for the currencies used most commonly. It use the sample image dataset to implement object recognition. We implement a model using tensor flow in python with a sample dataset of various classes to retrieve various elements in labelled images. The advancement we have found is the precision of finding the objects in images. It present Convolution neural networks for object recognition and classification in this paper. In conviction with other software, tools and APIs such as Tensor Flow Object Detection API, is an open source schema built on top of Tensor Flow that makes it easy to construct, train and deploy object detection models.

\section{BACKGROUND MOTIVATION}

As there are more than 150 different currencies available around the world so for the currency recognition the demand of an automated system has been exponentially growing. The need for constructing a system for processing notes with negligible human interference for various different uses had significance for the development of system which helps in detecting or identifying the paper notes. However this work becomes very critical due to the differing characteristics in each notes and the security measures involved in different currencies. Various methods were suggested like pattern matching to construct a system that uses a method for the recognition and identification of currencies. Not even a single technique has been proven to be sufficient enough for genuine improvement therefor making the issue an interesting area of research. In the early 90's proposal of an approach was made to identify the paper currency using image processing technique. It has been supposed that the notes taken were in good condition and the images are obtained as needed. It's critical to make reference that the system suggested need the input images which are taken in a predefined dimensions. The methodology discussed performs pre-processing steps in a sequence on input pictures and several features are then selected as hue, saturation and value criterion so that calculation of Euclidean distance using these parameters can be performed and this will be matched with the predefined parameters, set as standards. Though an algorithm was proposed in this system for the multiple currencies, but as the various notes across nations have similar features so this method is not an effective method to identify all the currencies.

\section{PROPOSED MODEL}

Our proposed system needs to be taught how an object or currency looks like in order to teach it to recognize that currency or object. We must teach the system how an rs10, rs20, rs100 etc. notes looks like before it can identify it. The precision of the system can be extended with the increase in number of labeled pictures the system takes to train the classifier. This type of learning is known as Supervised Learning. 


\section{International Journal of Engineering Applied Sciences and Technology, 2020 \\ Vol. 5, Issue 1, ISSN No. 2455-2143, Pages 539-542 \\ Published Online May 2020 in IJEAST (http://www.ijeast.com)}

Prior to starting with the preparation of system's model architecture, collecting proper data to train the model is an important step, in this case data refers to collection of several images of different categories which the system must recognize. A computer can only perform numerical computations but cannot interpret images as humans do. So in order to make the system understand the input, we need to convert the pictures into numbers or bytes.

Our object detection model has the following steps:

\section{A. Model Generation:-}

a. Dataset:-

The dataset include 7 classes which do not overlap with 200 images in every class. The images are perfectly labelled and are without any noise.

b. Splitting our dataset:-

It consumes very long period of time to calculate the gradient of model using a vast dataset as a whole. Therefore, during each iteration, a less number of images will be used. Then the dataset will be divided into $80 \%$ as image training set and $20 \%$ as image test set.

c. Pre-processing:-

We will perform the combination to the images randomly as mentioned. Cropping the images, horizontally flipping the images, resizing the images and convert the above dataset into required $\mathrm{xml}$ file.

d. Build a model:-

Convolutional Neural Networks (CNNS) are among the most popular techniques which used to enhance accuracy for images classification. It is a type of neural network that works similarly as a regular neural network, which would be the backbone of our model.

\section{B. Training the model:-}

A model is trained on the training dataset and tested on the validation set, and the model can be then improved over time over the validation set. The model can be applied to our actual test dataset, once we are satisfied with the results. Thus, on our validation set, we can see whether the model is over-fitting or under-fitting, which can help us to fit the model better and get better accuracy.

There are several prior models present in the tensor flow which we can use to train our dataset. We are using the faster R-CNN model to train our dataset.

Faster R-CNN is an object detection architecture which uses CNN like YOLO (You Look Only Once) and SSD (Single Shot Detector).

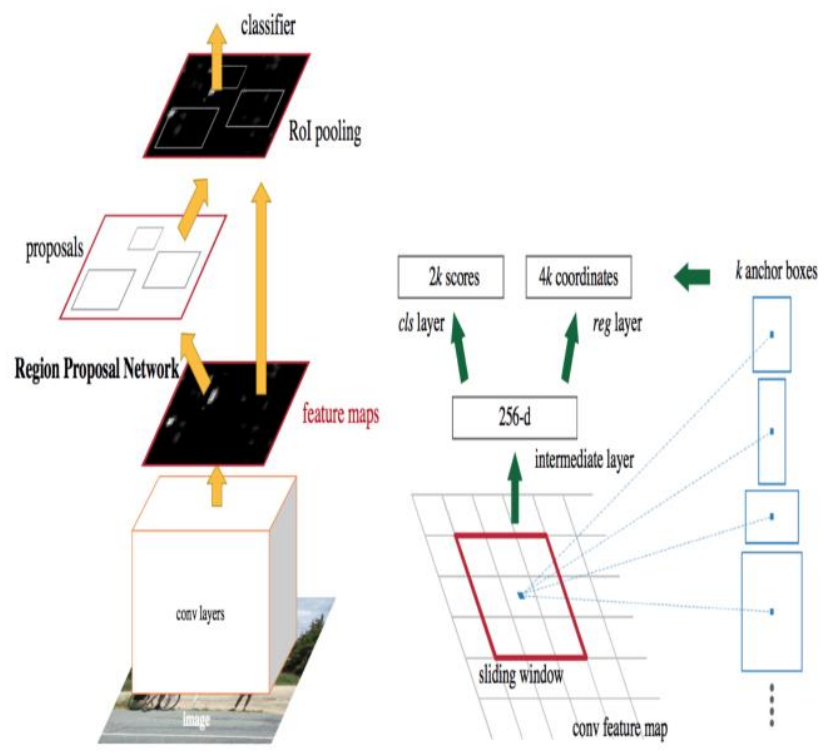

Fig. Faster R-CNN

It is made out of 3 sections:

1. Convolution layers:-

This layer is used in training the filters to extricate the images, for example let us assume that these filters are going to instructed in order to extract the suitable characteristics of a man face, then the filters that only existing in the human face are colors and shape of human face.

Convolution network are commonly made out of convolution layers, pooling layer and a last segment which is the completely associated or another expand things that will be utilized for a proper errand like characterization or identification.

We perform computation of convolution by adjusting the filters along our pictures given as input, the output is the matrix of two dimensions known as feature map. In the feature map the quantity of features will be decreased by eliminating the pixels having less intensity values, which is comprised in pooling. And the last step in Faster R-CNN is to arrange the features that do not belong to our case by utilizing the fully associated layers.

2. Region Proposal Network (RPN):-

RPN is a type of neural network based upon the final feature map of convolution layers and it also guess that whether an object present or not and those object's bounding boxes.

3. Classes and Bounding Boxes prediction:- 


\section{International Journal of Engineering Applied Sciences and Technology, 2020 \\ Vol. 5, Issue 1, ISSN No. 2455-2143, Pages 539-542 \\ Published Online May 2020 in IJEAST (http://www.ijeast.com)}

Now the range suggested by RPN will be given as an input to another completely associated neural network and speculate bounding boxes as well as object class.

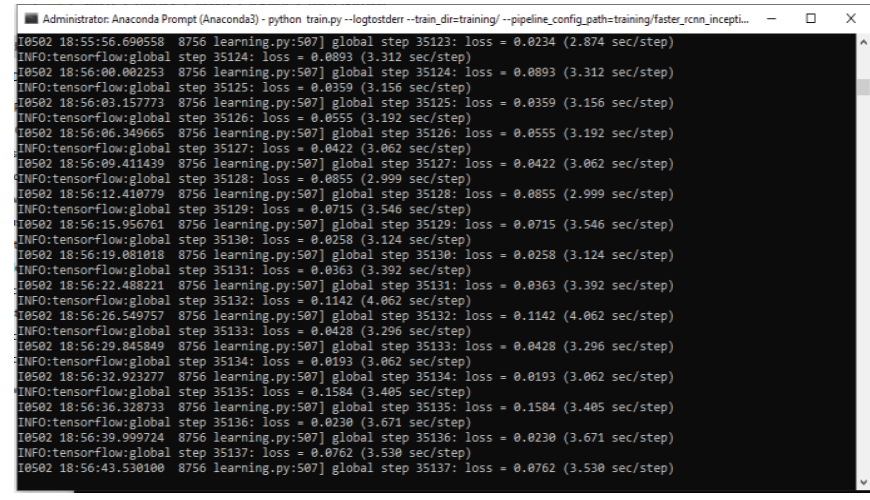

Fig . Model training

\section{Testing the model:-}

As we have trained our neural network on the custom dataset, now the model is ready to predict the output.

\section{RESULT}

To recognize the nation of currency, the banknotes have been segmented into the groups on the basis of blank region's presence and absence. Then, the banknotes will be classified by performing the template matching from the already stored templates that identifies the banknotes from the countries in the each group. For demonstration purpose, the Indian 500 rupee note is chosen. It is shown in below figures, before and after preprocessing steps.

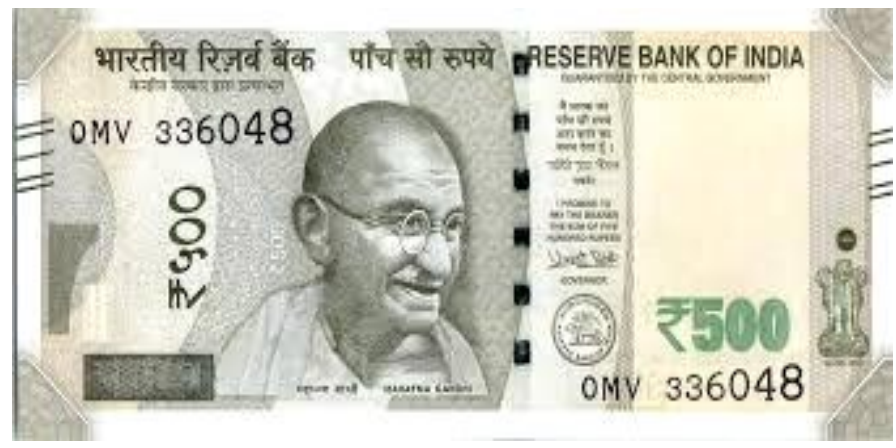

Fig. Original note

Once the image is done with preprocessing to recognize the country of origin of note, then we perform the classification on the templates of all the nations within its group.

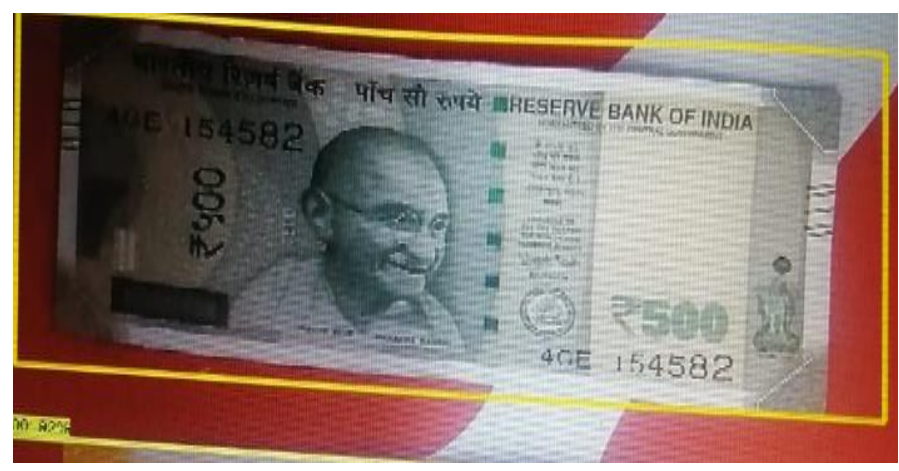

Fig. Processed image

Overall, it have been found that the accuracy of the system tends to be $93 \%$ i.e., the system is capable of identifying the cost of the notes as well as the country of origin of most of the notes accurately and correctly. This is a much better result.

\section{CONCLUSION}

A system have been developed for the accurate identification of country as well as the denomination of the note. The dataset upon which our system is trained, comprises of some of the most common currencies, but this dataset can easily be increased by using the same approach we have previously described. As observed that our system is capable to identify the denomination and country of the currency in approx. 5-6 seconds, which is considered as an improvement. As there are more than 150 currencies present in the world, but our system is concerned about few of the most commonly used currencies, but the number of different currencies to be recognized can be increased. Our system is not much efficient to recognize the damaged currencies which can be worked on in future.

\section{REFERENCE}

[1] Vedasamhitha Abburu "Currency Recognition System Prof. Sagar S.Rajebhosale", 2017, Tenth International Conference on Contemporary Computing (IC3) .

[2] Prof. Sagar S.Rajebhosale, March 2017, "Currency Recognition System Using Image Processing”, International Research Journal of Engineering and Technology (IRJET), Vol.1, Issue 03.

[3] Dr. Ritu Vijay, Vipin Kumar Jain, January 2013, Indian Currency Denomination Identification Using Image Processing Technique IJCSIT, Vol.4, issue 1,pp.126-128.

[4] Mahesh Pawar, Ketan Desai, "Object detection andCurrency Recognition using CNN", Cikitusi Journal for Multidisciplinary Research.

[5] Rubeena Mirza,Vinti Nanda,February 2012, Paper Currency verification System Based On characteristic 
Extraction Using Image Processing, IJEAT, Vol.1,Issue 03, pp.68-71.

[6] Padam Kumar, Harish Agarwal, “ Indian Currency Note Denomination Recognition in Color Image, Int. Journal on Advanced Computer Eng. AndCommunication Tech. Vol.1.

[7] JAmudhavel, Raswitha Bandi, Object Recognition Using Keras with Backend Tensor Flow.

[8] W. Li, Y. Li and K. Luo, 2008 "Application of image processing technology in paper currency classification system", Pattern Recognition 2008. CCPR '08. Chinese Conference on, pp. 1-5.

[9] F.Takeda , T. Nishikage, 2000 ,"Multiple kinds of paper currency recognition using neural network and application of Euro currency", IEEE International Joint Conference on Neural Networks, Como, pp. 143-147.

[10] Ms.Trupti Pathrabe and Dr. N.G.Bawane,2010, Paper Currency Recognition System Using Characteristics Extraction and Negativity Correlated NN Ensemble, Int. Journal of Latest Trends in Computing.

[11] Binod Prasad Yadav, 2014 "Indian Currency Recognition and VerificationSystem Using Image Processing". In: International Journal of Advanced Research in Computer Science and Software Engineering 4.12.

[12] Muhammad Sarfraz, 2015 "An Intelligent Paper Currency Recognition System”. In: Procedia Computer Science 65, pp. 538-545. 